\title{
La comunicación parental y la inteligencia emocional como desigualdad educativa en tiempo de Covid-19
}

\section{Parental communication and emotional intelligence as educational inequality in time of Covid-19}

1 Gissela Elizabeth Freire Cuello

Universidad Técnica de Ambato, Facultad de Ciencias de la Educación, Carrera de psicopedagogía. Ambato, Ecuador.

gfreire5840@uta.edu.ec

2 Roberto Enrique Alvarado Quinto

Universidad Técnica de Ambato, Facultad de Ciencias de la Educación, Carrera de psicopedagogía. Ambato, Ecuador

re.alvarado@uta.edu.ec

3 Fredy Aguilar Rodríguez

Universidad Técnica de Ambato, Facultad de Ciencias sociales, Carrera de comunicación.

Ambato, Ecuador.

wf.aguilar@uta.edu.ec

4 Rodrigo Andrade Alban

Universidad Técnica de Ambato, Facultad de Ciencias de la Educación, Carrera de

Psicopedagogía. Ambato, Ecuador

jr.andrade@uta.edu.ec

Artículo de Investigación Científica y Tecnológica

Enviado: 24/12/2021

Revisado: $29 / 12 / 2021$

Aceptado: $12 / 01 / 2022$

Publicado:08/03/2023

DOI: https://doi.org/10.33262/concienciadigital.v6i1.4.2057

Freire Cuello, G. E., Alvarado Quinto, R. E., Aguilar Rodríguez, F., \& Andrade Alban, R. (2023). La comunicación parental y la inteligencia emocional como desigualdad educativa en tiempo de Covid-19. ConcienciaDigital, 6(1.4), 1123-1131. https://doi.org/10.33262/concienciadigital.v6i1.4.2057

CONCIENCIA DIGITAL, es una Revista Multidisciplinar, Trimestral, que se publicará en soporte electrónico tiene como misión contribuir a la formación de profesionales competentes con visión humanística y crítica que sean capaces de exponer sus resultados investigativos y científicos en la misma medida que se promueva mediante su intervención cambios positivos en la sociedad. https://concienciadigital.org

La revista es editada por la Editorial Ciencia Digital (Editorial de prestigio registrada en la Cámara

Ecuatoriana de Libro con No de Afiliación 663) www.celibro.org.ec

Esta revista está protegida bajo una licencia Creative Commons Attribution Non Commercial No Derivatives 4.0 International. Copia de la licencia: http://creativecommons.org/licenses/by-nc-nd/4.0/ 
Palabras claves: educación, comunicación, pandemia, familia.
Keywords:

education, communication, pandemic, family.

\section{Resumen}

Las desigualdades académicas han sido un tema de mucha controversia durante la pandemia covid-19 y post covid-19 a nivel mundial; siendo un factor negativo para los niños, niñas y adolescentes cuyos padres no han adquirido toda su formación académica. Uno de los tipos de desigualdades educativas es la comunicación que mantienen los padres con los hijos debido a que esta se ha visto afectada al estar inmersos en un ámbito de estrés, ansiedad, impaciencia, poca tolerancia el cual les impide mantener una comunicación abierta, asertiva y afectiva esto también afecta en el ámbito educativo.

\begin{abstract}
Academic inequalities have been a topic of much controversy during the covid-19 and post covid-19 pandemic worldwide; being a negative factor for children and adolescents whose parents have not acquired all their academic training. One of the types of educational inequalities is the communication that parents maintain with their children because this has been affected by being immersed in an environment of stress, anxiety, impatience, low tolerance which prevents them from maintaining an open, assertive, and affective communication, this also affects the educational environment.
\end{abstract}

\section{Introducción}

Dentro del ámbito educativo las desigualdades académicas han sido un tema de mucha controversia durante la pandemia covid-19 y post covid-19 a nivel mundial; siendo un factor negativo para los niños, niñas y adolescentes cuyos padres no han adquirido toda su formación académica. Asimismo, el encontrar familias en un nivel de extrema pobreza ocasiona que los estudiantes mantengan desigualdades educativas al no contar con los recursos necesarios para ingresar a una educación virtual como se ha desarrollado durante la pandemia covid-19 (Mazas, 2019).

También otros factores han afectado a los procesos educativos como el psicológico, social y familiar. Durante la pandemia covid-19 la comunicación parental se ha visto influenciada directamente en la inteligencia emocional de los jóvenes Universitarios, al no existir una adecuada comunicación de padres a hijos, poca adaptabilidad en la dinámica familiar, el escaso manejo de estrés. De modo que la funcionalidad y roles parentales inciden de manera positiva o negativa en la familia y en los procesos 
educativos. La comunicación familiar es de gran importancia en la dinámica parental de padres e hijos en la que mantienen un vínculo afectivo con cada miembro de la familia, de igual manera dentro del hogar es en donde se establecen roles, reglas y esto da apertura en cuanto a la comunicación con el padre y madre cuyo objetivo es mantener una comunicación activa, asertiva, de calidad fuera de problemas o dificultades que se presenta en su hogar (Santos, 2019).

Los estilos de comunicación se dividen en agresivo, asertivo y pasivo, en cuanto al estilo de comunicación agresivo se basa generalmente en las personas que son egocéntricas en la que solo les importa el criterio de ellos y no de las demás personas de su alrededor; Las manifestaciones más comunes son agresiones verbales como insultos, tono de voz alta y amenazas constantes. De igual manera la comunicación asertiva es aquella que se da de forma directa en la que comunica la información de forma clara, precisa, concisa y afectiva sin que la comunicación llegue a ser ofensiva. Por consiguiente, la comunicación pasiva es en la cual la persona no refuta en ninguna opinión de los interlocutores sin respetar sus propios derechos al no comunicar sus deseos de forma libre y voluntaria, es por ello que no tiene una buena resolución de conflictos (Ruvalcaba \& Nava, 2018).

Los niveles de comunicación que se puede destacar dentro de la comunicación parental son:

La comunicación emotiva es aquella en la que se puede trasmitir los estados de ánimo, emociones, sentimientos y afecto. Al utilizar este tipo de comunicación la familia mantiene una interacción fluida entre los integrantes del hogar. De igual manera, la comunicación racional es aquella que se basa en las reflexiones personales en la que se pretende manipular la información y volverla formativa de tal manera que sirva para comunicar las normas y valores que deben seguir los hijos dentro y fuera de casa. Asimismo, la comunicación informativa es cuando se imparte información en forma general de los sucesos y hechos importantes dentro del vínculo familiar (Verde, 2019).

Dentro de la comunicación parental existen tres factores que influyen directamente como lo es la comunicación evitativa, comunicación ofensiva y la comunicación abierta. El factor de la comunicación evitativa se da por la carencia de compromiso para compartir sentimientos, ideas, criterios, ideologías, es por ello que no se establece una verdadera comunicación con fluidez entre padres e hijos. Asimismo, el factor de la comunicación ofensiva es aquella que se da por medio de la agresión verbal en la que afecta las relaciones intrapersonales e interpersonales con todos los miembros de la familia. Del mismo modo la comunicación abierta se basa en la interacción eficaz entre padres e hijos manteniendo empatía, escucha activa, armonía y fluidez semántica (Sánchez, 2018).

La inteligencia emocional es aquella que permite reconocer y canalizar las emociones que se ven reflejadas en la conducta implicando el uso de varias estrategias para afrontar los 
altos niveles emocionales que se presenta en el diario vivir. En la actualidad se evidencia altos niveles de estrés, ansiedad, pánico, miedo y rabia debido a la pandemia covid-19 que se ha venido presenciando en los dos últimos años; Desarrollar habilidades emocionales resulta un poco complejo debido a diversas situaciones de alto estrés que se encuentra expuesto a los integrantes de la familia por el hecho de permanecer todo el tiempo en casa sin tener interacción con la sociedad (Sierra, 2020).

Los principales componentes que se encuentran en la inteligencia emocional son:

El componente de la inteligencia intrapersonal se da por el reconocimiento de sí mismo, el asertividad, autoconcepto, autorrealización e independencia. De la misma forma el componente de la inteligencia interpersonal que se basa en mantener relaciones duraderas, teniendo capacidad de empatía y responsabilidad social para el cumplimiento de sus actividades diarias a favor de la sociedad. De igual manera, en el componente de adaptabilidad es aquel que se da por medio de la flexibilidad para adaptarse a nuevas situaciones, contextos y experiencias de la vida cotidiana. Asimismo, el componente del manejo del estrés es aquel que genera al control de impulsos para garantiza una óptima resolución de conflictos. Por último, el componente de estado de ánimo en general se presenta más en las personas con un alto nivel de optimismo cuyo propósito es la felicidad (Bar-On, 1997).

En definitiva, la comunicación parental y la inteligencia emocional se ven íntimamente relacionadas una con otra ya que mientas exista mayor comunicación entre padres e hijos mayor será la inteligencia emocional. Durante la pandemia covid-19 se ha venido dando diversos tipos de desigualdades educativas una de ellas es en la comunicación que mantienen los padres con los hijos debido a que esta se ha visto afectada al estar inmersos en un ámbito de estrés, ansiedad, impaciencia, poca tolerancia el cual les impide mantener una comunicación abierta, asertiva y afectiva esto también afecta en el ámbito educativo.

Es importante mantener una buena comunicación parental para que surja una adecuada estabilidad emocional en los jóvenes universitarios ya que los estudiantes dentro del ámbito educativo se pueden desempeñar de manera óptima si mantienen un adecuado manejo de emociones el cual les permite adaptase a los cambios existentes ocasionados por la pandemia covid-19.

Permanecer en una adecuación virtual sin mantener algún tipo de interacción social altera el estado de ánimo de los estudiantes es por eso que las emociones son de gran importancia dentro del aprendizaje ya que si existe mayor carga de emociones positivas mayor es la adquisición de información que esto se convierte en un aprendizaje significativo de largo plazo. 
Finalmente, la pandemia covid-19 ha afectado a la mayor parte de los estudiantes universitarios ocasionando desigualdades en el campo educativo debido a la falta de comunicación parental en donde existe una comunicación evitativa y ofensiva en la que padres e hijos están en constantes enfrentamientos, desacuerdos, poca tolerancia y desconfianza de tal manera que estos factores afectan a la estabilidad emocional de los estudiantes evidenciando una inadecuada tolerancia al estrés, carencia de control de impulsos y escasa resolución de conflictos.

\section{Metodología}

En la presente investigación se utilizó el enfoque mixto, de campo, descriptivo, documental bibliográfico de igual manera se utilizó una población de 150 estudiantes entre hombres y mujeres de la Universidad Técnica de Ambato en la Facultad de Ciencias Humanas y de la Educación en la carrera de psicopedagogía con una muestra de tipo aleatoria-simple.

Para la evaluación de la comunicación parental entre padres/hijo se utilizó la escala de comunicación Padres-Hijos/as (CA-M/CA-P) misma que consta de 40 ítems por los autores Barnes y Olson en 1997 y para evaluar la inteligencia emocional se utilizó el inventario de inteligencia emocional BarOn Ice mismo que consta de 133 ítems del autor Reuven BarOn. Los datos se obtuvieron por medio de la encuesta aplicada por Google Forms misma que se procesaron por Microsoft office Excel.

\section{Resultados}

\section{Figura 1}

\section{Escala de comunicación parental CA-M/CA-P}

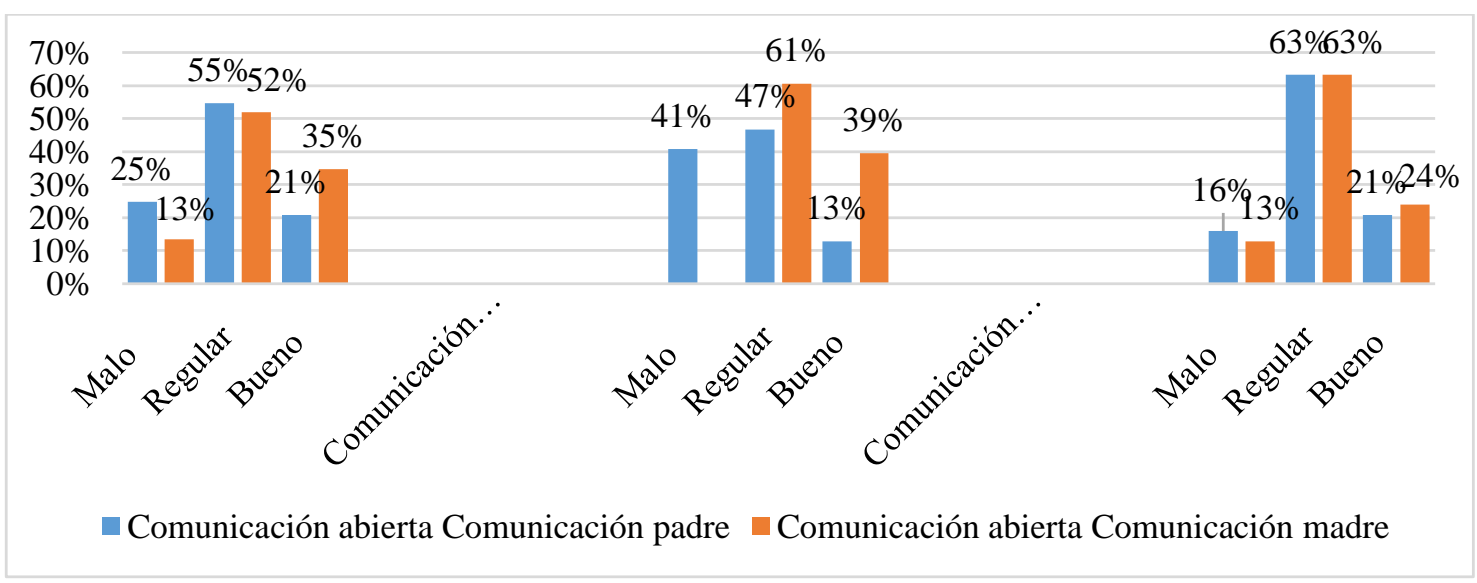

Nota: Distribución porcentual de los componentes del Cuestionario CM-CP

Fuente: Universidad Técnica de Ambato en estudiantes de la Carrera de Psicopedagogía 
Al obtener los resultados de la escala de comunicación parental CAM-CP se puede evidenciar que existe un porcentaje igualitarios del $63 \%$ en la comunicación evitativa tanto con el padre como la madre lo cual refiere que los estudiantes no resuelven los problemas de una manera adecuada y asertiva en la que los estudiantes universitarios no puedan llegar aún consenso con sus progenitores ya que evitan tener una conversación fluida sin ser juzgados por parte de sus padres de igual manera, huyen de los conflictos familiares. De igual manera, como lo menciona Cudris et al. (2020), en su artículo llamado Comunicación familiar en universitarios colombianos que existe un porcentaje del $80 \%$ en el parámetro bajo el cual refiere que los estudiantes presentan actitudes negativas y agresivas ante la figura paterna y materna ocasionando que no exista flexibilidad en el entorno familiar del mismo modo refieren la carencia de armonía en su hogar y fomentando una inadecuada resolución de conflictos.

\section{Figura 2}

\section{Cuestionario de inteligencia emocional BarOn Ice por componentes}



Nota: Distribución porcentual del Cuestionario de la Inteligencia Emocional BarOn ICE

Fuente: Universidad Técnica de Ambato en estudiantes de la Carrera de Psicopedagogía.

Como se puede evidenciar en los resultados obtenidos en el cuestionario e Inteligencia Emocional BarOn ICE en el componente de interpersonal con un porcentaje del $71 \%$ el cual hace referencia que los estudiantes presenta un alto nivel de empatía con sus familiares, pares y sociedad que los rodea, asimismo, mantienen una adecuada escucha activa, construyen relaciones amigables positivas que les permite mantener una adecuada 
capacidad emocional. Para Barrera-Gálvez et al. (2019), manifiesta en su artículo denominado La inteligencia emocional en estudiantes universitarios en el cual se puede evidenciar que presenta un porcentaje de $78 \%$ en el componente interpersonal en el marco de la empatía en la cual los estudiantes quienes fueron encuestados se encuentran en un en un estado emocional medio que refiere que en ocasiones socializan, comparten y entienden emociones de las personas que les rodea.

\section{Conclusiones}

- En la investigación realizada sobre la comunicación parental y la inteligencia emocional como una desigualdad educativa en tiempo de Covid-19, se realizó un análisis de los componentes investigados en donde se pudo evidenciar que los estudiantes de la Universidad Técnica de Ambato en tiempos de covid-19 presentan una comunicación parental de tipo regular con la ausencia de una comunicación abierta, en la que tanto padres e hijos no mantienen una adecuada comunicación asertiva generando desigualdades educativas ya que la mayor parte de los estudiantes refieren tener baja autoestima, desconfianza, enfrentamiento entre padres e hijos en la que los estudiantes no pueden expresarse de forma libre y voluntaria.

- De igual manera se pudo obtener datos reales en cuanto a la inteligencia emocional la que nos revela que los estudiantes mantienen independencia personal, alta capacidad de empatía, conocimiento de sí mismo; sin embargo, existe una carencia de manejo del estrés, control de impulsos y adaptabilidad en la que les cuesta ser flexibles a los cambios continuos, escasa resolución de conflictos; ocasionando una desigualdad educativa ya que mediante la falta de estos factores no se puede mantener un alto rendimiento académico.

\section{Referencias bibliográficas}

Bar-On, R. (1997). Emotional Quotient Inventory Technical Manual. Multi Health Systems.

Barrera-Gálvez, R., Solano-Pérez, C. T., Arias-Rico, J., Jaramillo-Morales, O. A., \& Jiménez-Sánchez, R. C. (2019). La inteligencia emocional en estudiantes universitarios. Educación y Salud Boletín Científico Instituto de Ciencias de la Salud Universidad Autónoma del estado de Hidalgo, 7(14), 50-55.

Cudris Tores, L., Gutiérrez García, R., Barrios Núñez, A., Manjarrez Hernández, M., \& Pérez Corzo, E. (2020). Comunicación familiar en universitarios colombianos. 
Mazas, J. (2019). (In)movilidad social y vulnerabilidad: Un estudio en torno a la reproducción social en. Universidad de Cantabria.

Ruvalcaba, N. O., \& Nava, J. M. (2018). Relaciones escolares, comunicación con padres y prosocialidad como predictores de emociones positivas. Liberabit: Revista Peruana de Psicología, 24(2), 183-93.

Sánchez Narvasta, G. (2018). Comunicación Parental en las estudiantes del Segundo de secundaria de la Institución Educativa No 20335. Nuestra Señora del Carmen, Huaura.

Santos, J. (2019). Comunicación en familias nucleares con hijos adolescentes. Revista Científica Digital de Psicología, 8, 1-11.

Sierra, S. (2020). Inteligencia emocional y práctica clínica en tiempos de pandemia. CES Medicina, 34, 59-68.

Verde Torres, Y. R. (2019). Comunicación familiar prevalente en estudiantes de una institución educativa, Chimbote.

\section{Cliencia Digital}


El artículo que se publica es de exclusiva responsabilidad de los autores y no necesariamente reflejan el pensamiento de la Revista Conciencia Digital.

\section{Ciencia}

El artículo queda en propiedad de la revista y, por tanto, su publicación parcial y/o total en otro medio tiene que ser autorizado por el director de la Revista Conciencia Digital.
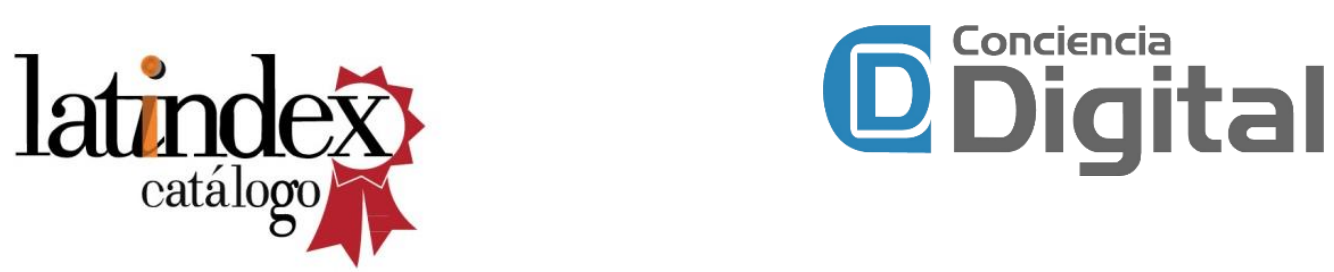

Indexaciones

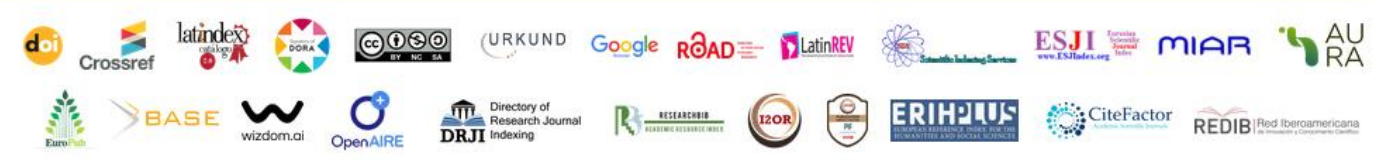

